\title{
Tree Routing Improvement Algorithm Based on the Neighbor Table in ZigBee Networks
}

\author{
Leqiang Bai \\ Information \& Control Engineering Faculty \\ Shenyang Jianzhu University \\ Shenyang, China \\ e-mail: baileqiang@sjzu.edu.cn \\ Jingjing Sun \\ Information \& Control Engineering Faculty \\ Shenyang Jianzhu University \\ Shenyang, China \\ e-mail: sjjwang402@163.com
}

\author{
Jing Dai \\ Information \& Control Engineering Faculty \\ Shenyang Jianzhu University \\ Shenyang, China \\ e-mail: daijing6615@163.com \\ Ye Tian \\ Information \& Control Engineering Faculty \\ Shenyang Jianzhu University \\ Shenyang, China \\ e-mail: t3jds@126.com
}

\begin{abstract}
Aiming at the problems of ZigBee tree routing algorithm that the algorithm has many hop counts, long latency of data transmission and low ratio of the packets delivery, a tree routing improvement algorithm based on the neighbor table in ZigBee networks is proposed. The proposed algorithm establishes strategy of neighbor nodes selection with the aid of 1-hop neighbor nodes address information and selects the neighbor node which has the minimum tree hop counts as the next hop forwarding node. When the hop count is the same, the improvement algorithm selects the node which has high LQI value as the next hop forwarding node. The simulation results show that the improvement algorithm could reduce the number of forwarding nodes efficiently, improve the reliability of the network data transmission and achieve the purpose of improving the network performance.
\end{abstract}

Keywords-ZigBee networks; address assignment; neighbor table; LQI; tree routing

\section{INTRODUCTION}

ZigBee networks technology is a kind of wireless network technology which has the characteristics of short distance, low consumption, low cost, low complexity, self-organizing low data transmission features and so on[1]. The cluster-tree network is supported by the IEEE 802.15.4/ZigBee [2]. ZigBee networks technology is mainly used in industry, agriculture, military, medical, and intelligence Home Furnishing environment.

The AODVjr routing algorithm is aimed at AODV algorithm's [3]improvement and can find the shortest routing path from the source node to the destination node by a routing discovery process in [4], but node needs to maintain a routing table in the process of searching a routing path in the AODVjr routing algorithm, and requires very high resources. The treeing routing algorithm is mentioned in [5]. It only relies on the relevant node's parent nodes or child nodes to select a routing path, and does not need the participation of the routing table. Based on the above characteristics, it effectively saves the network storage resources. But using this algorithm, the data packets is always transfer to the first common ancestors node of the source node and the destination node's firstly and forwards to the destination node with a larger routing path cost. The ITRA routing algorithm, which uses neighbor node address information is mentioned in [6], can select less hop counts of the routing path. The algorithm does not need nodes to store routing table information, saving more energy than the AODVjr algorithm. But the ITRA algorithm does not consider whether the selected hop counts are the minimum tree hop counts or not, and whether multiple transmission paths is existed from the source node to the destination node or not. The improvement algorithm is mentioned in [7], which can find a better path with the aid of the neighbor table. But this algorithm does not consider whether it has multiple transmission paths in the process of choosing the next hop node, calculates all neighbor nodes to the destination node of hop counts, and selects the node that has the minimum tree hop counts among neighbor nodes to transmit packets, leading to an increasing of workload.

In view of the above problems, this paper proposes a tree routing improvement algorithm based on the neighbor table in ZigBee networks.

\section{TREe Routing ALgORITHM ANALYSIS}

ZigBee networks nodes equipment mainly can be divided into: coordinator, routing equipment and end devices [1].

\section{A. Address Assignment}

The ZigBee network uses a distributed address assignment mechanism to assign a unique network address for each of the network equipment. $\mathrm{C}_{\mathrm{m}}$ stands for the maximum number of the children nodes, $R_{m}$ stands for the maximum number of the children router nodes, $\mathrm{L}_{\mathrm{m}}$ stands for the maximum network level [1]. Cskip (d) represents address offset which is distributed by the parent nodes to 
their child nodes. If a node's level is $d$ and its address is $A_{\text {parent }}$, then the address $A_{n}$ of its nth child router node is:

$$
A_{\mathrm{n}}=A_{\text {parent }}+C_{\text {skip }}(d) \times(n-1)+1
$$

The address $A_{m}$ of its mth end devices node is:

$$
A_{\mathrm{m}}=A_{\text {parent }}+C_{\text {skip }}(d) \times R_{m}+m
$$

$\mathrm{C}_{\text {skip }}$ (d) value is computed by formula:

$$
C_{\text {skip }}(d)= \begin{cases}1+C_{m} \times\left(L_{m}-d-1\right) & , R_{m}=1 \\ \frac{1+C_{m}-R_{m}-C_{m} \times R_{m} L_{m}-d-1}{1-R_{m}}, & R_{m} \neq 1\end{cases}
$$

\section{B. Neighbor Table}

The neighbor table and neighbor node are provided by the ZigBee protocol [1].In the process of building the ZigBee networks, each ZigBee device module will be in the form of broadcast to exchange with its 1-hop node information. By transmitting information with each other, any node can know the basic information of all nodes in 1hop ranges, and the stores the information in its corresponding neighbor list. That is to say two nodes can directly communicate with each other in 1-hop ranges. According to the needs of users, artificially adding some basic information in the neighbor table of contents is possible, such as Link Quality Indication (LQI) value and so on.

LQI is used to represent an index of energy and quality of the received data frames. The size of LQI is based on the signal intensity and the detected signal to noise ratio. The IEEE802.15.4 standard defines LQI as received frame strength and/or quality characteristics and limits LQI value in the $0 \sim 255$ ranges. A higher LQI value can indicate a better link quality, a better transmission performance and a more reliable link [8].

\section{Tree Routing Algorithm}

In the tree routing algorithm, network nodes calculate the next hop according to the network address of the destination node [9]. When a node which its depth is d and address is A transmits data to the destination address $\mathrm{D}$, the tree routing algorithm judges node $\mathrm{A}$ of descendant nodes whether have the destination node or not. If the destination node is the node A's descendant node, the packets are forwarded to the corresponding child node. Determining the destination node $\mathrm{D}$ is node $\mathrm{A}$ of descendant node that needs to satisfy the conditions:

$$
A<D<A+C_{\text {skip }}(d-1)
$$

\begin{tabular}{|c|c|}
\hline Symbol & Definition \\
\hline $\mathrm{A}$ & Network node address \\
\hline $\mathrm{D}$ & Destination node address \\
\hline $\mathrm{A}_{\mathrm{i}}, \mathrm{i}=1,2, \ldots, \mathrm{I}$ & $\begin{array}{l}\text { The current node's parent or child } \\
\text { node address }\end{array}$ \\
\hline $\mathrm{A}_{\mathrm{j}}, \mathrm{j}=\mathrm{I}+1, \mathrm{I}+2, \ldots, \mathrm{J}$ & $\begin{array}{l}\text { The current node's neighbor node } \\
\text { address except for its parent or } \\
\text { child node }\end{array}$ \\
\hline $\mathrm{M}_{\mathrm{k}}, \mathrm{k}=1,2, \ldots, \mathrm{J}-\mathrm{I}$ & $M_{k=}=D-A_{j}$ \\
\hline $\mathrm{TRC}(\mathrm{A}, \mathrm{D})$ & The tree hop count from A to D \\
\hline B & $\begin{array}{c}\min \operatorname{TRC}\left(\mathrm{A}_{\mathrm{j}}, \mathrm{D}\right) \text { corresponds to the } \\
\text { neighbor node set } \mathrm{B}\end{array}$ \\
\hline $\mathrm{C}$ & $\begin{array}{c}C=\left\{A_{i}, B\right\} \\
A_{i} \text { satisfy min TRC }\left(A_{i}, D\right)\end{array}$ \\
\hline
\end{tabular}

Under the premise of formula (4), the next hop node address $\mathrm{N}$ of the node $\mathrm{A}$ is as follows formula (5):
TABLE I. AlgorithM SYMBOL DEFINITION

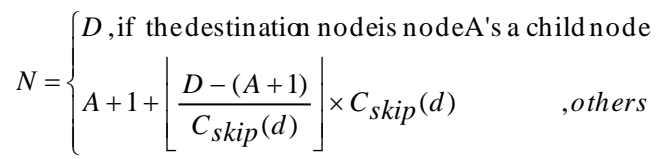

If the descendant node $\mathrm{D}$ is not the descendant node of node $\mathrm{A}$, then according to the tree structure, forward the packets to the parent node of node A [10].

In ZigBee networks, based on ZigBee address allocation mechanism, the tree routing algorithm can get common ancestors node with the maximum depth of the source node and the destination node. The network depth is represented by MaxDepth. Using DstDepth, SrcDepth and HopCount respectively represent the network depth of the destination node, the network depth of the source node and routing hop counts. According to the tree routing algorithm, routing hop counts can be calculated by formula (6) [5].

$$
\text { Hop Count }=\text { DstDepth }+ \text { SrcDepth }-2 \times \text { MaxDepth }
$$

\section{IMPROVED ALGORITHM DESIGNING}

With the aid of neighbor table, the algorithm can further reduce the hop count, avoid unnecessary waste of network nodes, consider the node LQI values and improve the performance of network. The algorithm is denoted the received packets' node as the current node.

Neighbor nodes selection strategy:

The current node uses $\left|\mathrm{D}-\mathrm{A}_{\mathrm{j}}\right|$ to calculate the value of $\mathrm{M}_{\mathrm{k}}$ successively, and then the value of $\mathrm{M}_{\mathrm{k}}$ are sorted from small to large, that is $\left(\mathrm{M}_{1}, \mathrm{M}_{2}, \ldots, \mathrm{M}_{\mathrm{k}}, \ldots, \mathrm{M}_{\mathrm{J}-\mathrm{I}}\right)$.

Based on ZigBee address allocation mechanism, we search the maximum depth common ancestors node of $\left(\mathrm{M}_{1}, \mathrm{M}_{2}, \ldots, \mathrm{M}_{\mathrm{k}-1}, \mathrm{M}_{\mathrm{k}}, \ldots, \mathrm{M}_{\mathrm{J}-\mathrm{I}}\right)$ corresponds to the neighbor node and destination node successively, when the maximum depth common ancestors node of the neighbor node and destination node address is 0 ,which means the node is coordinator, it stop searching. If $\mathrm{M}_{\mathrm{k}}$ corresponds to this neighbor nodes, according to ZigBee address allocation mechanism, we can obtain the maximum depth common ancestors nodes of $\left(\mathrm{M}_{1}, \mathrm{M}_{2}, \ldots, \mathrm{M}_{\mathrm{k}-1}\right)$ corresponds to the neighbor node and destination node, which are not coordinator, however, according to ZigBee address allocation mechanism, we can obtain the maximum depth common ancestors nodes of $\left(\mathrm{M}_{\mathrm{k}}, \ldots, \mathrm{M}_{\mathrm{J}-\mathrm{I}}\right)$ corresponds to 
the neighbor node and destination node, which are coordinator. According to the characteristics of the tree topology, we can know the tree routing hop counts of $\left(\mathrm{M}_{\mathrm{k}}, \ldots, \mathrm{M}_{\mathrm{J}-\mathrm{I}}\right)$ corresponds to the neighbor node to destination node are larger than the tree routing hop counts of $\left(\mathrm{M}_{1}, \mathrm{M}_{2}, \ldots, \mathrm{M}_{\mathrm{k}-1}\right)$ corresponds to the neighbor node to destination node.

The improvement algorithm's steps are as follows:

1) The current node judges whether itself is the destination node or not.

If the current node itself is the destination node, the current node will receive packets; otherwise it turns to 2)

2) The current node judges whether 1-hop neighbor nodes of it have the destination node or not.

If 1-hop neighbor nodes of the current node have the destination node, it receives the packets; otherwise it turns to 3 ).

3) According to the tree structure, the current node seeks a node from node $A_{i}$ which has the minimum tree hop counts to the destination node.

a) The current node uses the formula (4)to judge whether the descendant nodes of it have the destination node or not.

If the descendant nodes of current node have the destination nodes, using the formula (5) to select the corresponding child node $\mathrm{A}_{\mathrm{i}}$ that arriving to the destination node has the minimum tree hop counts. According to ZigBee address allocation mechanism, we search the maximum depth common ancestors node of the corresponding child node $\mathrm{Ai}$ and destination node, and it uses the formula (6)to calculate the value of $\operatorname{TRC}\left(\mathrm{A}_{\mathrm{i}}\right.$, D), and turns to 4); otherwise turns to $b$ ).

b) According to tree structure, it selects the current node's the parent node Ai as the node which has the minimum tree hop counts to the destination node. According to ZigBee address allocation mechanism, we search the maximum depth common ancestors nodes of the current node's the parent node and destination node. It uses the formula (6)to calculate the value of TRC $\left(A_{i}\right.$, D), and turns to 4$)$.

4) The node $A_{j}$ uses the formula (6)to calculate the value of $\operatorname{TRC}\left(A_{j}, D\right)$, then forms a set of $B$ which is consisted by the min $\operatorname{TRC}\left(A_{j}, D\right)$ corresponds to the neighbor nodes.

a) Finding the neighbor node which corresponds to $\left(M_{1}, M_{2}, \ldots, M_{J-I}\right)$ successively.

b) According to ZigBee address allocation mechanism, we search the maximum depth common ancestors node of $M_{1}$ corresponds to the neighbor node and the destination node, and judge whether the maximum depth common ancestors nodes is coordinator or not.

If the maximum depth common ancestors node of $\mathrm{M}_{1}$ corresponds to the neighbor node and destination node is coordinator, it uses the formula (6) to calculate the hop counts of $\left(\mathrm{M}_{1}, \mathrm{M}_{2}, \ldots, \mathrm{M}_{\mathrm{J}-\mathrm{I}}\right)$ corresponds to the neighbor node to destination node successively until nodes $A_{j}$ are all evaluated, it forms a set of $\mathrm{B}$ which is consisted by the $\min \operatorname{TRC}\left(\mathrm{A}_{\mathrm{j}}, \mathrm{D}\right)$ corresponds to the neighbor nodes , and turns to 5); If the maximum depth common ancestors node of $\mathrm{M}_{1}$ corresponds to the neighbor node and destination node is not coordinator, it search the maximum depth common ancestors node of $\left(\mathrm{M}_{2}, \ldots, \mathrm{M}_{\mathrm{J}-\mathrm{I}}\right)$ corresponds to the neighbor node and destination node successively until the maximum depth common ancestors node address is coordinator or nodes $\mathrm{A}_{\mathrm{j}}$ are all evaluated. All eligible neighbor nodes use the formula (6) to calculate hop counts to the destination node successively, it forms a set of $B$ which is consisted by the $\min \operatorname{TRC}\left(\mathrm{A}_{\mathrm{j}}, \mathrm{D}\right)$ corresponds to the neighbor nodes, and turns to 5$)$.

5) Comparing $\min \operatorname{TRC}\left(A_{j}, D\right)$ with $\operatorname{TRC}\left(A_{i}, D\right)$, and choosing the node set which has the minimum tree hop counts to the destination node.

a) When $\min \operatorname{TRC}\left(A_{j}, D\right)=\operatorname{TRC}\left(A_{i}, D\right)$, set $C$ is selected, then turns to 6$)$.

b) When min $\operatorname{TRC}\left(A_{j}, D\right)>\operatorname{TRC}\left(A_{i}, D\right)$, node $A_{i}$ is selected as the next hop forwarding node and is received the transmit packets.

c) When min $\operatorname{TRC}\left(A_{j}, D\right)<\operatorname{TRC}\left(A_{i}, D\right)$, set $B$ is selected, then turns to 6$)$.

6) In the set calculated by step 5), the node of the maximal LQI value is selected as the next hop forwarding node and is received the transmit packets.

The algorithm follows the principle of the minimum tree hop counts to select the next hop forwarding node, until the packets have been sent to the destination node.

\section{The Results Of Simulation AND ANALYsis}

To verify the performance of improvement algorithm, the three aspects of the average hop count, end-to-end latency from the source node to the destination node and transmission success rate are simulated. The improvement algorithm is compared to the tree routing algorithm and ITRA algorithm [6]. This paper uses MATLAB platform to simulate the improvement algorithm, tree routing algorithm and ITRA algorithm. The simulation parameters: selecting $\mathrm{Cm}=6, \mathrm{Rm}=6, \mathrm{Lm}=4$, and the ranges built by the formula (1), (2) and (3)is $100 \mathrm{~m} \times 100 \mathrm{~m}$, node number is 300 , maximum transmission distance is $25 \mathrm{~m}$ ZigBee network. Where the packets size is 80 bytes, the types of data flow are CBR, the rate of sending packets is 1 packets/s and the simulation time is set to $200 \mathrm{~s}$. The experiment selects 6 size scenes whose number of the nodes is from 50 to 300 to simulate each algorithm. Each scene of simulation data was calculated the average after independent operation 100 times.

As shown in figure 1, with the increasing number of network nodes, the hop count of the node transmission data is increasing, and the average hop count of the improvement algorithm is less than the tree routing algorithm and ITRA algorithm. Improvement algorithm considers the information of neighbor nodes address and by using the neighbor node selection strategy to select to the destination node of hop counts for the less, as the next hop forwarding node in the neighbor nodes of the current node, making data transmission path further optimized. 


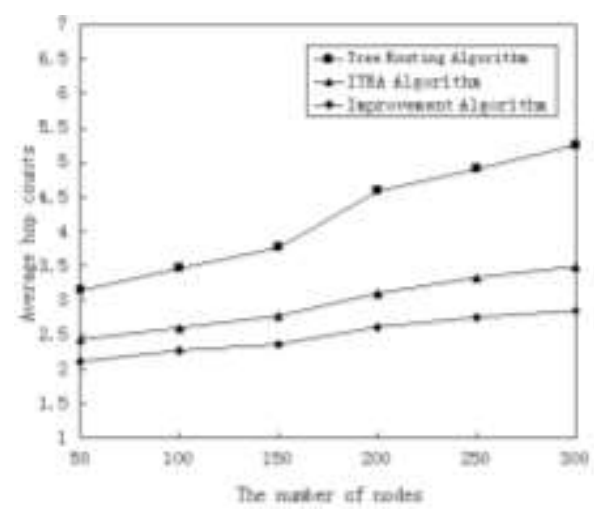

Figure 1. The average hop count

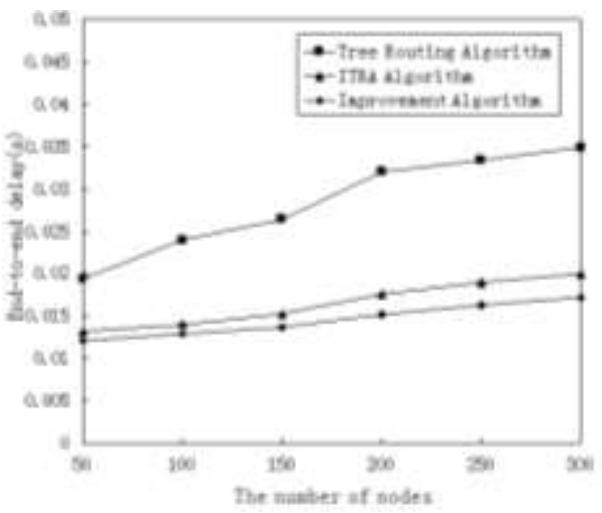

Figure 2. End-to-end latency

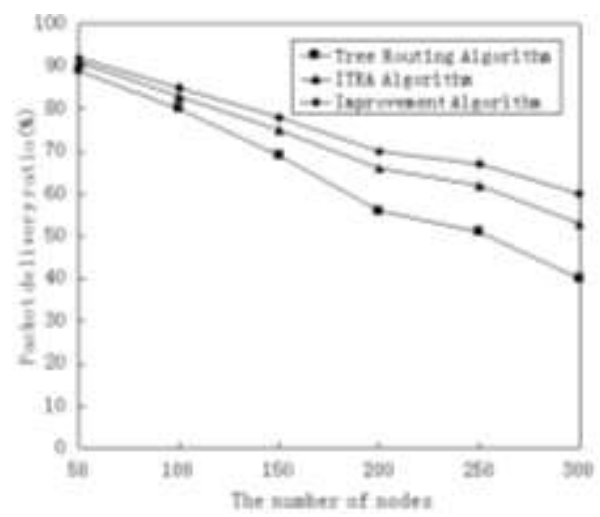

Figure 3. Packet delivery ratio

As shown in Fig.2, the end-to-end latency is mainly affected by the hop counts between the source node and the destination node. With the increasing number of network nodes, the latency of the improvement algorithm from the source node to the destination is less than the tree routing algorithm and the ITRA algorithm. Reducing algorithm's the routing hop counts, can effectively reduce the latency of the source node to destination node and achieve the purpose of network performance improvement.

As shown in figure 3 , as density of the network nodes increases, signal interference becomes stronger, and the hop count of the transmission path are more increase, the number of data packets from the source node to the destination node successfully in the improvement algorithm are higher than the tree routing algorithm and ITRA algorithm. Whether the packets delivery ratio is high or not, it is mainly affected by the degree of interference, and the transmission routing path hop counts. Improvement algorithm considers neighbor node LQI value factors, and selects the maximum LQI value of the node as the next hop forwarding node, further improving the packets delivery ratio.

\section{CONCLUSIONS}

This paper proposed a tree routing improvement algorithm based on the neighbor table in ZigBee networks. The algorithm uses node information in the neighbor table and the characteristics of the tree topology, and selects the node that has the minimum tree hop counts as next hop forwarding node in the 1-hop neighbor nodes. When the hop count is the same, the node whose LQI value is the maximum is selected as the next hop forwarding node. Simulation results show that the improvement algorithm effectively reduces hop counts and network data transmission latency, and improves the reliability of the network data transmission. The algorithm does not need to store the routing table and is applicable to the resource constraints of wireless sensor networks.

\section{ACKNOWLEDGMENT}

This research was financially supported by the natural science foundation of Liaoning Province (201102180).

\section{REFERENCES}

[1] ZigBee Alliance. ZigBee Specification, 2009.

[2] Zden`ek Hanzálek, Member, IEEE, and Petr Juřcík. Energy Efficient Scheduling for Cluster-Tree Wireless Sensor Networks with Time-Bounded Data Flows: Application to IEEE 802.15.4/ZigBee. IEEE Transactions on Industrial Informatics VOL. 6, NO. 3, AUGUST 2010:438-450.

[3] Jun Wang · Min Chen· Victor C.M. Leung. Forming priority based and energy balanced ZigBee networks - a pricing approach. Telecommun Syst 2013:1281-1292.

[4] HE Lingling. An Improved Cluster-Tree Routing Algorithm in ZigBee Networks. Chinese Journal of Sensors and Actuators, 2010, 23(9), pp.1303-1307.

[5] Taehong Kim, Seong Hoon Kim, Jinyoung Yang, Seong-eun Yoo, Member, IEEE, and Daeyoung Kim, Member, IEEE. Neighbor Table Based Shortcut Tree Routing in ZigBee Wireless Networks. IEEE Transactions on Parallel and Distributed Systems, 2014 pp.706-716.

[6] QI Jian-chao, WEI Zhen. An improved tree-based routing algorithm for ZigBee. Journal of Hefei University of Technology, 2010, 33(4), pp.529-532,537.

[7] LI Gang, CHEN Jun-jie, GE Wen-tao.An Improved Cluster-Tree Routing Algorithm in ZigBee Networks. Observation and Control Technology, 2009, 28(9):52-55.

[8] JIANG Ting, ZHAO Cheng Lin. Purple Mountain Technology and Its Application [M]. Beijing:Beijing University of Posts and Telecommunications Publishing House, 2006.

[9] Gang Ding, Student Member, IEEE, Zafer Sahinoglu, Senior Member, IEEE ,Philip Orlik, Member, IEEE, Jin Yun Zhang, Senior Member, IEEE, and Bharat Bhargava, Fellow, IEEE. TreeBased Data Broadcast in IEEE802.15.4 and ZigBee Networks. IEEE Transactions on Mobile Computing, 2006, pp.1516-1574.

[10] YAO Yu-kun, LI Peng-xiang, REN Zhi, GU Yuan. Borrowed address assignment algorithm for ZigBee network. Journal of Computer Applications, 2011, 31(8), pp.2044-2047,2064 